\title{
Ansiedade-traço competitiva: diferenças quanto ao gênero, faixa etária, experiência em competições e modalidade esportiva em jovens atletas
}

\author{
Marina Pereira Gonçalves - Universidade Federal do Rio Grande do Norte ${ }^{1}$ \\ Raquel Pereira Belo - Universidade Federal da Paraíba
}

\begin{abstract}
Resumo
O presente estudo se propõe a investigar a ansiedade-traço competitiva no que diz respeito ao gênero, faixa etária, experiência em competições e tipo de modalidade esportiva (individual e coletiva) em jovens atletas. Para sua realização, participaram 105 atletas paraibanos de diferentes modalidades esportivas, com idades variando de 11 a 20 anos e a maioria do sexo masculino. Estes, em seus próprios locais de treino, responderam à Escala de AnsiedadeTraço Competitiva e questões sociodemográficas. Com base na análise dos dados, os resultados indicaram que as mulheres apresentaram maior ansiedade-traço competitiva do que os homens, não se encontrando diferença significativa entre as outras variáveis estudadas. Sugere-se a realização de pesquisas futuras com a utilização de outros instrumentos e considerando novas variáveis.

Palavras-chave: Ansiedade-traço competitiva; Jovens atletas.
\end{abstract}

\section{Competitive trait-anxiety: differences of gender, age group, experience in competitions and modality sport in young athletes}

\begin{abstract}
This study aimed to investigate the anxiety in competition situations, considering the gender, age groups, sportive modality and the experience of the athletes. To the accomplishment of this research, participated 105 athletes from Paraíba, of different sportive modalities, being most male and with ages varying between 11 and 20 years. These athletes, in his own training places, answered Sport Competition Anxiety Trait and socio-demographic questions. From the data analysis, the results showed that the women presented greater competitive trait-anxiety than the men. It suggested the accomplishment of researches on the future that use other scales and variables.
\end{abstract}

Keywords: Competitive trait-anxiety; Young athletes.

\section{Introdução}

É inegável o papel significativo que o esporte e o jogo recreativo têm em qualquer cultura ou sociedade em todo o mundo, capaz de colocar em cena as emoções, os sentimentos, os esforços, o tempo e a energia, de uma forma que nenhuma outra atividade se aproxima. Entretanto, os diversos elementos que compõem o cenário esportivo podem tanto auxiliar quanto prejudicar o desempenho do atleta, chegando em alguns casos a provocar emoções não apenas positivas, mas negativas também. Nesse cenário, agressão, ansiedade, estresse, frustração, medo, auto-superação, realização, prazer, alegria e divertimento, aparecem entrelaçados como os principais sentimentos associados à prática esportiva (Dobránszky \& Machado, 2001).

$\mathrm{Na}$ literatura da psicologia aplicada ao esporte, a ansiedade é vista como uma das principais variáveis que interferem no desempenho dos atletas (Figueiredo, 2000). A maioria dos esportistas sofre pressão, medo e ansiedade causada pela obrigação de vencer, algo característico em uma sociedade na qual exalta a emoção da vitória e o sofrimento da derrota (Bara Filho \& Miranda, 1998).

A espera pela "lista de corte" de um time ou o jogo de estréia de um time infanto-juvenil em um campeonato mundial podem ser citados como exemplos de situações geradoras de ansiedade nos atletas. A exposição a esses tipos de experiências ansiosas pode muitas vezes causar mudanças fisiológicas como o estreitamento da atenção, o aumento no consumo de energia, o aumento de adrenalina, o que interfere negativamente no desempenho global do atleta (Martin, 1996, citado por Figueiredo, 2000). Observa-se ainda que as competições esportivas podem ser vistas como situações ameaçadoras, capazes de gerar variados níveis de ansiedade nos atletas. Gill e Deeter (1988) multidimen-

${ }^{1}$ Endereço para correspondência:

Rua Senador José Ferreira de Souza, 1.937 - apto 201 - Candelária - 59064-520 - Natal-RN

E-mail: marinapgoncalves@gmail.com 
sionam a competitividade entre atletas com tendências comportamentais que podem ser classificadas em competidor (reflete o prazer de competir em qualquer situação); determinado (caracterizado por estabelecer objetivos com desejos pessoais que podem ser de vitória ou de preparação para o desenvolvimento pela experiência); e vitorioso (busca a vitória como único objetivo em comparação interpessoal). Assim, alguns competidores ávidos por vencer podem superar desafios e ter suas melhores performances em competições difíceis, enquanto atletas mais apreensivos podem evitar desafios, se sentir mais pressionados e ter um desempenho abaixo das expectativas. Dessa maneira, fica claro perceber que, dependendo da forma como o atleta interpreta as situações competitivas como ameaçadoras ou não, poderão ocorrer alguns efeitos negativos no seu desempenho. Um deles é a ansiedade.

Para Spielberger (1972), a ansiedade é um complexo estado ou condição psicológica do organismo humano, constituída por propriedades fenomenológicas e fisiológicas que se diferencia de estados emocionais como o estresse, a ameaça e o medo, pois tais eventos se apresentam como possíveis causadores do estado de ansiedade. Além disso, a ansiedade pode se apresentar de formas distintas como, por exemplo, a ansiedade enquanto estado ou enquanto traço. $\mathrm{O}$ estado de ansiedade (A-estado) refere-se a um estado emocional transitório ou condição do organismo humano caracterizado por sentimentos desagradáveis de tensão e apreensão conscientemente percebidos, e por um aumento na atividade do sistema nervoso autônomo, gerando reações psicofisiológicas como taquicardia, "frio na barriga", "arrepio na espinha", entre outras. Por outro lado, o traço de ansiedade (A-traço) refere-se a diferenças individuais relativamente estáveis em propensão à ansiedade. Em geral, seria de se esperar que os indivíduos que apresentam alta A-traço demonstrariam elevações de A-estado, pois se a circunstância for percebida como ameaçadora, sem objeto de perigo, ou seja, simbólica, inespecífica e antecipada, o indivíduo responde com alta ansiedadeestado. Porém, se a circunstância for percebida como não ameaçadora, o indivíduo reage com ansiedadeestado baixa (Spielberger, 1972). Mas, se pessoas que diferem em A-traço mostrarão ou não diferenças correspondentes em A-estado, depende do grau em que a situação específica é percebida por um indivíduo em particular como perigosa ou ameaçadora, e isso é grandemente influenciado por experiências passadas do indivíduo (Biaggio, Natalício \& Spielberger, 1977).

A teoria da ansiedade traço-estado de Spielberger (1972) serviu de base a inúmeras pesquisas na área esportiva. Os investigadores passaram a adotá-la na avaliação da ansiedade no desporto utilizando o STAI (Spielberger, Gorsuch \& Lushene, 1970), que no Brasil é conhecido por IDATE - Inventário de Ansiedade Traço-Estado (Biaggio e cols., 1977). O IDATE compreende duas escalas paralelas, uma para medir a ansiedade enquanto traço (IDATE-T) e outra para medir a ansiedade enquanto estado (IDATE-E). Entretanto, os investigadores perceberam a necessidade de se ter medidas específicas de ansiedade no desporto, de modo que com o desenvolvimento desses instrumentos, o IDATE passou a ser menos utilizado no contexto esportivo e substituído por outros instrumentos (Dias, 2005).

Dessa forma, baseado nos estudos de Spielberger (1972), Martens (1977), desenvolveu a Teoria da Ansiedade-Traço Competitiva, que seria a predisposição de perceber situações competitivas como ameaçadoras, representando um indicativo de como um atleta reagiria ao interpretar certas situações competitivas como ameaçadoras ao seu bem-estar físico, psicológico e social. Para medir a ansiedade-traço competitiva em atletas, Martens, Vealey e Burton (1990a) desenvolveram um questionário específico conhecido por Sport Competition Anxiety Test - SCAT, que foi o primeiro questionário desenvolvido com base no pressuposto de que uma escala de traço de ansiedade específica do desporto seria um melhor preditor da ansiedade-estado em situações competitivas do que uma escala geral de ansiedade.

No entanto, ainda carecia de uma escala da ansiedade-estado específica do desporto, de modo que Martens e cols. (1990a) desenvolveram o Competitive State Anxiety Inventory - 2 (CSAI-2), que mede a ansiedadeestado competitiva a partir de três subescalas (ansiedadeestado cognitiva, a ansiedade-estado somática e a autoconfiança). Martens e cols. (1990) sugerem a utilização do SCAT antes de fazer uso do CSAI-2, já que o SCAT identifica uma predisposição característica para a ansiedade-traço competitiva. Desde então, as duas medidas têm sido utilizadas com freqüência em pesquisas nacionais e internacionais sobre ansiedade no contexto esportivo, algumas delas apresentadas a seguir.

No Brasil, algumas pesquisas foram realizadas utilizando o SCAT para identificar ansiedade-traço competitiva em atletas, principalmente no que diz respeito às diferenças de gênero, faixa etária e modalidade esportiva (individuais ou coletivos). De Rose Júnior e Vasconcellos (1997), por exemplo, contando com a participação de atletas entre 10 e 16 anos, buscaram estabelecer comparações quanto às diferentes faixas etárias (categoria pré-mirim, mirim e infantil) e sexos em relação à ansiedade-traço competitiva. Tais autores encontraram diferenças estatisticamente

Psico-USF, v. 12, n. 2, p. 301-307, jul./ dez. 2007 
significativas entre os níveis de ansiedade-traço competitiva dos homens e mulheres, onde estas apresentaram níveis mais elevados. Em relação às idades, foram encontradas na categoria infantil dos homens e mulheres diferenças significativas estatisticamente.

Ademais, Detanico e Santos (2005), em um estudo com 122 atletas judocas participantes dos Jogos Abertos de Santa Catarina em 2004, também utilizando o SCAT, identificaram que as mulheres judocas mostraram-se mais ansiosas que os homens e que a ansiedade dos judocas tem associação com a idade, já que quanto maior a idade, menos ansiosos os judocas se mostraram. Entretanto os níveis de ansiedade-traço competitiva apresentados pelos judocas não foram influenciados pelo tempo de prática dos mesmos.

Becker Jr. (2000), analisando os estudos de Martens (1977), identificou níveis diferenciados de ansiedade quanto à estrutura do esporte: individual ou coletivo. Nos estudos citados pode-se observar que atletas de esporte individual apresentam maior nível de ansiedade do que os de equipe, especificamente na natação e no atletismo (talvez em decorrência de cada atleta saber que não há como se recuperar de uma má saída). Além disso, em esportes individuais os atletas não compartilham a responsabilidade, expondo-se sozinhos a uma avaliação direta dos expectadores, gerando uma pressão desproporcional à demonstração de excelência na performance.

Outro estudo realizado sobre a ansiedade e o tipo de esporte (individual ou coletivo) foi o de Zeng (2003), que buscou comparar vários níveis de ansiedade e autoconfiança entre atletas de esportes individuais e coletivos com idades entre 18 e 23 anos. Os resultados indicaram que os atletas de esportes coletivos têm menores níveis de ansiedade-estado cognitiva e ansiedade-estado somática quando comparados com atletas de esportes individuais. Entretanto, o nível de ansiedade-traço competitiva entre atletas de esporte coletivo e individual foram semelhantes. Acrescenta-se que os atletas de esporte coletivo têm altos níveis de estado de autoconfiança, estado de confiança esportiva e traço de confiança esportiva quando comparados com atletas de esporte individual.

Alguns pesquisadores têm investigado a relação da ansiedade com outros construtos presentes no contexto esportivo. Por exemplo, com o propósito de investigar a relação entre estresse, ansiedade competitiva, estados de humor e suporte social em danos atlético, Lavallé e Flint (1996) realizaram um estudo com jogadores de futebol e de rugby do sexo masculino, com idades entre 19 e 28 anos de uma universidade canadense. Os resultados revelaram que a ansiedadetraço competitiva, através do SCAT, foi significativa-

Psico-USF, v. 12, n. 2, p. 301-307, jul./ der. 2007 mente correlacionada com os fatores de tensão/ ansiedade e raiva /hostilidade, bem como com o escore total dos estados de humor negativos. Além disso, acrescenta-se que os índices de danos dos atletas foram correlacionados significativamente com o SCAT.

Dando continuidade a esse tipo de investigação, Dias (2005) analisou as relações entre ansiedade, estresse, emoções e confronto no contexto esportivo, contando com a participação de 550 atletas de diversas modalidades e de ambos os sexos, de categorias juvenil, júnior e sênior de 46 clubes diferentes em Portugal. Os resultados demonstraram que, em comparação com os atletas do sexo masculino, as atletas do sexo feminino pareciam exibir níveis significativamente mais elevados de ansiedade e percepção de ameaça, além de recorrer a estratégias de confronto menos eficazes. Em relação à modalidade, os atletas das modalidades individuais apresentaram níveis mais elevados de ansiedade e percepção de ameaça do que os das modalidades coletivas, e os atletas mais jovens (juniores/juvenis) revelaram empregar estratégias menos eficazes do que os da categoria sênior.

Dunn e Causgrove Dunn (2001) realizaram um estudo para verificar a relação do SCAT e do Sport Anxiety Scale - SAS (Smith, Smoll, \& Schutz, 1990) com as quatro subescalas do Collegiate Hockey Worry Scale CHWS (Dunn, 1999): a medida de esporte específica que mede tendências dos atletas para preocupar-se sobre o fracasso no desempenho, a avaliação social negativa, a avaliação de perigo físico e a avaliação das situações de incerteza. Com uma amostra de 178 jogadores de hockey no gelo, os resultados indicam que há uma relação entre preocupar-se com o fracasso e ter uma avaliação social negativa com a ansiedade-traço competitiva, não havendo relação do SCAT e do SAS com o perigo físico e situações de incerteza. Com base nesses resultados, os autores apontam a necessidade de desenvolvimento de instrumentos com subescalas medindo componentes situacionais diferentes da ansiedade-traço competitiva.

A ansiedade também pode ser medida em pessoas envolvidas no contexto esportivo, não apenas nos atletas, como por exemplo, no estudo de Burke, Joyner, Pim e Czech (2000) com 25 oficiais de basquete de escolas e faculdades do Sudeste da Georgia (EUA) que, utilizando uma versão modificada do SCAT e do CSAI-2 para oficiais, compararam suas respostas com as normas do SCAT para jogadores de basquete. Os resultados demonstraram que os oficiais apresentaram médias significativamente menores do que a média dos jogadores de basquete durante um torneio. Quanto aos resultados do CSAI-2, os oficiais relataram sentir menos ansiedade cognitiva após o torneio do que antes. A 
quantidade e variedade de estudos realizados sobre a ansiedade-traço competitiva revelam a necessidade de se verificar como a mesma se apresenta, de modo que as investigações não devem deixar de ser realizadas.

Diante de tais observações, o presente estudo se propõe a investigar a ansiedade-traço competitiva no que diz respeito ao gênero, faixa etária, experiência em competições e tipo de modalidade esportiva (individual e coletiva) em jovens atletas, contribuindo de alguma forma para a produção do conhecimento científico sobre ansiedade no contexto esportivo.

\section{Método}

\section{Delineamento}

O presente estudo é de tipo correlacional, contando com medidas de natureza ex post facto. Tem como objetivo comparar a ansiedade-traço competitiva com variáveis como idade, gênero, modalidade esportiva e nível de competição.

\section{Amostra}

Participaram do presente estudo 105 atletas paraibanos, advindos de diversas modalidades esportivas (Futsal - 14,3\%; Handbol - 5,7\%, Nado sincronizado - 9,5\%; Natação - 33,3\% e Vôlei $37,1 \%)$, com idades variando de 11 a 20 anos $(M=15,23$ anos e $\mathrm{DP}=1,76)$ sendo a maioria do sexo masculino $(55,2 \%)$. Todos os dados foram coletados na cidade de João Pessoa (PB).

\section{Instrumentos}

Escala de Ansiedade-traço competitiva (Sport Competition Anxiety Test-SCAT):

Originalmente desenvolvida por Martens e cols. (1990), adaptada e traduzida por De Rose Júnior e Rosamilha (1985), citados por De Rose Júnior (1985), é composta por quinze itens indicadores de ansiedadetraço competitiva. Esses itens são respondidos numa escala de tipo Likert de 3 pontos, onde $\mathrm{A}=$ dificilmente; $\mathrm{B}=$ às vezes e $\mathrm{C}=$ sempre, na qual o sujeito deve expressar o grau de concordância com relação a cada frase. Martens e cols. (1990) apresentam com detalhes o processo de validação do SCAT incluindo evidências de consistência interna (KR-20 com valores variando de $0,95$ a 0,97$)$, com alta fidedignidade teste-reteste $(0,77)$ e uma lista de 80 estudos publicados que têm utilizado o SCAT como instrumento de medida da ansiedade-traço competitiva.

\section{Caracterização da amostra}

Foram elaboradas questões de caráter sociodemográfico (por exemplo, idade, sexo, renda) e outras que permitissem avaliar a experiência dos atletas em competições esportivas, bem como o tipo de modalidade (individual ou coletiva).

\section{Procedimento}

Para a coleta dos dados foram recrutados aplicadores de ambos os sexos, que se dirigiam aos locais de treino dos atletas (clubes, colégios, etc.) pedindo permissão aos técnicos para a realização da pesquisa. Todos os instrumentos eram de auto-aplicação, exigindo o mínimo de interferência por parte do seu aplicador. Todas as respostas foram dadas individualmente, e, antes que respondessem, os participantes eram informados sobre o caráter confidencial de suas respostas. Em caso de falta de compreensão sobre como responder ou alguma outra questão de forma, é que os aplicadores auxiliaram os respondentes.

\section{Análise dos dados}

A versão 13 do pacote estatístico SPSSWIN (Statistical Package for the Social Sciences) foi utilizada para a tabulação e análise dos dados. Além dos indicadores descritivos (média, desvio padrão, etc.), foi realizada uma análise de variância (ANOVA) para comparar as médias entre os grupos estudados.

\section{Resultados}

A fim de verificar as médias dos grupos estudados quanto à ansiedade-traço competitiva, foi realizada uma ANOVA (teste Post Hoc de Bonferroni), tendo como variável dependente a ansiedade-traço competitiva e como variáveis independentes o gênero, a faixa-etária, a experiência em competições e o tipo de modalidade esportiva (individual ou coletiva) dos atletas. Os resultados podem ser vistos na Tabela 1.

Os resultados indicaram que houve diferença estatisticamente significativa da ansiedade-traço competitiva em relação ao gênero dos participantes $[F$ $(295,08)=16,85 ; \mathrm{p}<0,000)$. Analisando a Tabela 1, podese observar que segundo o teste de Bonferroni essa diferença indicou que as mulheres apresentaram maior ansiedade-traço competitiva $(\mathrm{M}=33,42 ; \mathrm{DP}=0,87)$ do que os homens $(\mathrm{M}=28,78 ; \mathrm{DP}=0,72)$. Entretanto, em relação à faixa etária, não foi observada diferença estatisticamente significativa $[F(0,01)=0,01 ; \mathrm{p}<0,97]$, ocorrendo o mesmo no que se refere a experiência em competições $[F(25,36)=1,45$; $\mathrm{p}<0,23]$ e ao tipo de modalidade esportiva $[F(45,58)=$ $\mathrm{p}<0,11]$. 
Tabela 1 - Média e desvio padrão da ansiedade-traço competitiva em função do gênero, faixa etária, experiência em competições e tipo de modalidade esportiva

\begin{tabular}{|c|c|c|}
\hline \multirow{2}{*}{ Grupos } & \multicolumn{2}{|c|}{ Ansiedade-traço competitiva } \\
\hline & M & $\mathrm{DP}$ \\
\hline \multicolumn{3}{|l|}{ Gênero } \\
\hline Masculino $(n=58)$ & 28,78 & 0,72 \\
\hline \multirow[t]{2}{*}{ Feminino $(n=47)$} & 33,42 & 0,87 \\
\hline & $\begin{array}{c}\mathrm{F}(295,08)=16,85 \\
\mathrm{p}<0,000\end{array}$ & \\
\hline \multicolumn{3}{|l|}{ Faixa etária } \\
\hline $11-14$ anos $(n=61)$ & 30,92 & 0,75 \\
\hline \multirow[t]{3}{*}{$15-20$ anos $(n=44)$} & 30,89 & 0,84 \\
\hline & $\mathrm{F}(0,01)=0,00$ & \\
\hline & $\mathrm{p}<0,97$ & \\
\hline \multicolumn{3}{|l|}{ Experiência em competições } \\
\hline Local $(n=12)$ & 33,93 & 1,68 \\
\hline Estadual $(n=16)$ & 29,41 & 1,06 \\
\hline Norte-nordeste $(\mathrm{n}=33)$ & 30,23 & 1,03 \\
\hline Nacional $(\mathrm{n}=37)$ & 30,66 & 1,03 \\
\hline \multirow[t]{3}{*}{ Internacional $(n=7)$} & 31,89 & 1,80 \\
\hline & $F(25,36)=1,45$ & \\
\hline & $\mathrm{p}<0,23$ & \\
\hline \multicolumn{3}{|l|}{ Modalidade Esportiva } \\
\hline Individual $(n=35)$ & 30,89 & 0,66 \\
\hline \multirow[t]{3}{*}{ Coletiva $(n=70)$} & 30,92 & 0,97 \\
\hline & $F(45,58)=2,60$ & \\
\hline & $\mathrm{p}<0,11$ & \\
\hline
\end{tabular}

\section{Discussão e conclusão}

O esporte competitivo apresenta particularidades como confronto, demonstração, comparação e avaliação constante de seus participantes, fazendo da competição uma situação na qual o desenvolvimento e a performance do atleta sejam sempre comparados com algum padrão já existente (De Rose Júnior \& Vasconcellos, 1997). Esse tipo de situação gera ansiedade, sendo uma das variáveis mais estudadas no contexto esportivo. Assim, estudar os diversos níveis de ansiedade em relação às competições, considerando o gênero, a idade, a experiência dos atletas, bem como o tipo de modalidade esportiva, foi o objetivo do presente estudo.

As diferenças de gênero quanto à ansiedade-traço competitiva, coincidem com o que se verifica na literatura, a qual indica que as mulheres apresentam maior ansiedade competitiva do que os homens. Segundo alguns autores (De Rose Júnior \& Vasconcellos, 1997; Dias, 2005; La Rosa, 1998), isso pode estar relacionado com o fato das competições ainda serem enfatizadas para atletas do sexo masculino, fazendo que a mulher, ao competir, seja muito mais cobrada: o que explicaria os índices mais elevados de ansiedade diante de esportes de competição. Além disso, pode-se também levar em consideração a cultura na qual o atleta está inserido. Dessa forma, sugere-se aqui que tais resultados possam ser mais bem investigados a fim de uma maior compreensão a respeito da participação da mulher nas diferentes modalidades esportivas, bem como nas diferentes regiões do país.

Com relação às faixas etárias e à ansiedade-traço competitiva, não houve diferença estatisticamente significativa. Este resultado não corrobora estudos anteriores (De Rose Júnior \& Vasconcellos, 1997; Detanico \& Santos, 2005), que indicam que os atletas mais jovens e, provavelmente, com menos vivências e experiência no esporte de competição, seriam mais vulneráveis a demonstrar ansiedade no contexto esportivo do que os atletas mais velhos e, portanto, mais independentes, mais experientes e com estratégias de enfrentamento mais elaboradas quanto a situações ansiosas no esporte. Apesar da influência da idade não ter sido verificada no presente estudo, é importante ressaltar que os treinadores, os pais, e todos os outros profissionais envolvidos com o cenário esportivo devem considerar a idade dos atletas, pois, como visto na literatura, os mais 
jovens podem ainda não se encontrar tão preparados diante de muitas cobranças e pressões do esporte de competição (De Rose Júnior \& Vasconcellos, 1997; Gill e Deeter, 1988).

No que se refere à experiência dos atletas em competições, não se encontrou diferença significativa quanto à ansiedade-traço competitiva. Esperava-se que o atleta que já houvesse participado de competições em nível internacional, nacional e norte-nordeste possuiria um pouco mais de experiências em competições do que aquele que houvesse participado apenas de competições locais e estaduais, o que diminuiria seu nível de ansiedade. Entretanto, deve-se destacar que no presente estudo considerou-se o nível da competição como um possível indicador da experiência dos atletas diante das competições, porém, sugere-se que em novos estudos outras formas de investigação considerando esta mesma variável devam ser realizadas, tendo em vista que, por exemplo, alguns atletas possam ter participado de um número considerável de competições locais, enquanto outros possam ter participado de poucas competições em nível nacional, não se podendo, neste caso, afirmar qual dos dois atletas teria mais experiência em competições, sendo esta uma das limitações deste estudo.

Finalmente, os resultados encontrados entre a ansiedade-traço competitiva e o tipo de modalidade esportiva também não apresentaram índices estatisticamente significativos, não estando de acordo com estudos anteriores (Becker Jr., 2000, Martens, 1977; Zeng, 2003). Os referidos estudos demonstram que os atletas apresentam menores índices de ansiedade nos esportes coletivos, onde a presença dos companheiros de equipe diminuiria a responsabilidade individual diante dos resultados das competições, explicando a maior elevação da ansiedade dos atletas em esportes individuais.

Apesar de não se ter encontrado os resultados esperados entre as variáveis estudadas, pode-se dizer que os participantes deste estudo indicaram alguns níveis diferenciados de ansiedade-traço competitiva, demonstrando que, no geral, a ansiedade faz parte do cotidiano dos atletas paraibanos, mas a mesma se manifesta em maior ou menor grau, dependendo de inúmeros fatores, principalmente quanto ao gênero dos participantes.

Não se descartam possíveis limitações neste estudo, tais como a não-representatividade de todas as variáveis de forma equilibrada e a verificação apenas da ansiedade enquanto traço, de modo que a ansiedade enquanto estado e a consideração de outras variáveis como, por exemplo, a participação dos pais, a influência dos técnicos e da torcida, a influência da ansiedade no abandono dos atletas ao esporte, a consideração de esportes específicos na determinação da ansiedade-traço competitiva, entre outros, são indicados para estudos futuros.

De forma geral, alguns resultados são coerentes com os existentes na literatura, o que sugere a pertinência da presente pesquisa. A ansiedade faz parte do cotidiano de diversas pessoas e, em diferentes momentos de suas vidas, os atletas deparam-se com ela a todo instante. Assim, conhecer como a ansiedade se expressa em relação a diferentes variáveis que envolvem o contexto esportivo é de suma importância para que se criem estratégias de intervenção eficazes, pois os atletas constituem um público específico que necessita ser "cuidado", respeitando-se as suas reais condições e estilo de vida.

\section{Referências}

Bara Filho \& Miranda (1998). Aspectos psicológicos do esporte competitivo. Treinamento Desportivo, 3(3) 6272.

Becker Jr., B. (2000). Manual de psicologia do esporte e exercicicio. Porto Alegre, RS: Nova Prova.

Biaggio, A. M. B., Natalício, L. \& Spielberger, C. D. (1977). Desenvolvimento da forma experimental em português do Inventário de Ansiedade Traço-Estado (IDATE) de Spielberger. Arquivos Brasileiros de Psicologia, 19(3) 31-44.

Burke, K., Joyner, A. B., Pim, A. \& Czech, D. R. (2000). An exploratory investigation of the perceptions of anxiety among basketball officials before, during, and after the contest. Journal of Sport Behavior, 23(1), 11-19.

De Rose Júnior, D. (1985). Influência do grau de ansiedadetraço no aproveitamento de lances-livres. Dissertação de Mestrado. São Paulo: Universidade de São Paulo Escola de Educação Física.

De Rose Júnior, D. \& Vasconcellos, E. G. (1997). Ansiedade-traço competitiva e atletismo: um estudo com atletas infanto-juvenis. Revista Paulista de Educação Física, 11(2), 148-157.

Detanico, D. \& Santos, S. G. (2005). Variáveis influenciando e sendo influenciadas pela ansiedadetraço competitiva: um estudo com atletas judocas. Lecturas: Educación Física y Deporte [On-line], 10(90). Obtido em 20 de junho 2007 do World Wide Web: http://www.efdeportes.com/.

Dias, C. S. L. (2005). Do stress e ansiedade às emocões no desporto: da importância da sua compreensão à necessidade da sua gestão. Tese de Doutorado. Portugal: Universidade do Minho.

Dobránszky, I. de A. \& Machado, A. A. (2001). Autoeficácia: um estudo da sua contribuição para a 
avaliação de desempenho de atletas. Revista PsicoUSF, 6(2) 67-74.

Dunn, J. G. H. (1999). A theoretical framework for structuring the content of competitive worry in ice hockey. Journal of Sport \& Exercise Psychology, 21, 259279.

Dunn, J. G. H. \& Causgrove Dunn, J. (2001). Relationships among the Sport Competition Anxiety Test, the Sport Anxiety Scale, and the Collegiate Hockey Worry Scale. Journal of Applied Sport Psychology, 13(4), 411-429.

Figueiredo, S. H. (2000). Variáveis que interferem no desempenho do atleta de alto rendimento. Em K. Rubio (Org.). Psicologia do esporte: interfaces, pesquisa e intenvenção (pp. 114-124). São Paulo: Casa do Psicólogo.

Gill, D. L. \& Deeter, T. E. (1988). Development of the sport orientation questionnaire. Research Quartely for Exercise and Sport, 59(3), 191-202.

La Rosa, J. (1998). Ansiedade, sexo, nível sócioeconômico e ordem de nascimento. Psicologia: Reflexão e Crítica, 11(1), 59-70.

Lavallé, L. \& Flint, F. (1996). The relationship of stress, competitive anxiety, mood state, and social support to athetic injury. Journal of Atbletic Training, 31(4), 296299.

Martens, R. (1977). Sport Competition Anxiety Test. Champaing: Human Kinetics.
Martens, R., Vealey, R. S. \& Burton, D. (1990). Competitive anxiety in sport. Champaign, IL: Human Kinetics.

Martens, R., Burton, D., Vealey, R. S., Bump, L. A. \& Smith, D. E. (1990). Development and validation of the Competitive State Anxiety Inventory - 2. Em R. Martens, R. S. Vealey \& D. Burton (Eds.). Competitive anxiety in sport (pp. 117-232). Champaign, IL: Human Kinetics.

Smith, R. E., Smoll, F. L. \& Schutz, R. W. (1990). Measurement and correlates of sportspecific cognitive and somatic trait anxiety: The Sport Anxiety Scale. Anxiety Research, 2, 263-280.

Spielberger, C. D. (1972). Anxiety: cursent trends in theory and research. New York: Academic Press.

Spielberger, C. D., Gorsuch, R. L. \& Lushene, R. E. (1970). Idate: Inventário de Ansiedade Traço-Estado. Rio de Janeiro: Cepa.

Zeng, H. Z. (2003). The difference between anxiety and self-confidence between team and individual sports college varsity athletes. Internacional Sports Journal, Winter, 28-34.
Recebido em março de 2007 Reformulado em agosto de 2007 Aprovado em setembro de 2007

Sobre as autoras:

Marina Pereira Gonçalves é psicóloga formada pela Universidade Federal da Paraíba e mestranda em Psicologia pela Universidade Federal do Rio Grande do Norte. Possui trabalhos apresentados em eventos locais, regionais, nacionais e internacionais na área da Psicologia Social, Psicometria e Psicologia do Esporte. Atualmente tem pesquisado principalmente sobre motivação à prática de atividades físicas, como ênfase na adaptação e validação de instrumentos psicológicos no contexto esportivo.

Raquel Pereira Belo é psicóloga pela Universidade Federal de Pernambuco e mestre em Psicologia Social pela Universidade Federal da Paraíba. Atualmente é aluna do Programa de Pós-Graduação em Psicologia Social - Nível Doutorado na mesma instituição. No presente estudo - monografia de conclusão do curso de graduação em Psicologia - participou como orientadora da primeira autora enquanto professora substituta também na Universidade Federal da Paraíba. 
